\title{
FORCED VIBRATIONS OF PIEZOELECTRIC CRYSTAL PLATES*
}

\author{
BY \\ H. F. TIERSTEN AND R. D. MINDLIN \\ Columbia University
}

1. Introduction. In a previous paper [1] (referred to in the sequel as $I$ ), Cauchy's two-dimensional equations of coupled flexural and extensional motion of crystal plates were extended to the next higher order of approximation so as to accommodate the two lowest thickness-shear modes. In the present paper a further extension is made to include the piezoelectric relations and the electric field equations. The equations obtained are also extensions of previous equations [2] in which the thickness-shear modes, the piezoelectric effect and the electric field equations were taken into account, but coupling with extensional modes was omitted. The new equations are deduced from the threedimensional, linear, piezoelectric equations by a procedure based, as in $I$, on the series expansion methods of Cauchy and Poisson and the variational method of Kirchhoff. The theorems of uniqueness and orthogonality established in $I$ for the approximate equations are extended to include coupling with the electric field. The solution of problems of steady, forced vibration is considered and is reduced to the solution of the associated free vibration problem plus some quadratures. The case of a plate driven by an ac-voltage applied to electrodes on its faces is investigated and a formula is given for the total surface charge. An application is made to the forced vibrations of rectangular, rotated $Y$-cut quartz plates.

2. Three-dimensional equations. The three-dimensional equations, from which the plate equations will be deduced, are: the variational forms of the equations of motion and electrostatics; the strain-displacement and electric field-potential relations; and the linear, piezoelectric constitutive relations. They may be written, respectively, as

$$
\begin{gathered}
\int_{R}\left(T_{i, i}-\rho u_{j, t \imath}\right) \delta u_{i} d R=0, \quad \int_{R} D_{i, i} \delta \varphi d R=0 \\
S_{i j}=\frac{1}{2}\left(u_{i, j}+u_{j, i}\right), \quad E_{i}=-\varphi_{, i}, \\
T_{i j}=c_{i j k l}^{E} S_{k l}-e_{k i j} E_{k}, \quad D_{i}=e_{i k l} S_{k l}+\epsilon_{i k}^{S} E_{k},
\end{gathered}
$$

where $T_{i j}, u_{i}, D_{i}, S_{i i}, E_{i}$ are the components of stress, mechanical displacement, electric displacement, strain and electric field, respectively; $\rho, R$ and $\varphi$ are the mass density, volume and electric potential, respectively; and $c_{i j k l}^{E}, e_{k i j}, \epsilon_{i k}^{S}$ are the elastic, piezoelectric and dielectric constants, respectively. We employ rectangular coordinates $x_{i}, i=1,2,3$, and the summation convention for repeated tensor indices. A comma followed by an index or $t$ denotes differentiation with respect to a space coordinate or time, respectively.

3. Expansion in power series. The faces of the plate are taken at $x_{2}= \pm h$ and the remaining boundary is a cylindrical surface with generators perpendicular to the

\footnotetext{
*Received September 25, 1961.
} 
faces. We expand $u_{i}$ and $D_{i}$ in a series of powers of the thickness-coordinate:

$$
u_{i}=\sum_{n=0}^{g} x_{2}^{n} u_{j}^{(n)}, \quad D_{i}=\sum_{n=0}^{o} x_{2}^{n} D_{i}^{(n)}+\delta_{2 i} x_{2}^{o+1} D_{2}^{(0+1)},
$$

where $g$ is a positive integer, $\delta_{i j}$ is the Kronecker delta and the variables $u_{i}^{(n)}, D_{i}^{(n)}$ are independent of $x_{2}$. When the series expressions are substituted in (2.1) and the integrations with respect to $x_{2}$ are performed, the result is

$$
\begin{gathered}
\int_{A} \sum_{n=0}^{o}\left(T_{i j, i}^{(n)}-n T_{2 i}^{(n-1)}+F_{i}^{(n)}-\rho \sum_{m=0}^{g} H_{m n} u_{j, t t}^{(m)}\right) \delta u_{i}^{(n)} d A=0, \\
\int_{A} \sum_{n=0}^{o}\left(D_{i, i}^{(n)}+(n+1) D_{2}^{(n+1)}\right) \delta \varphi^{(n)} d A=0,
\end{gathered}
$$

where $A$ is the area of the plate and

$$
\begin{gathered}
T_{i i}^{(n)} \equiv \int_{-h}^{h} x_{2}^{n} T_{i i} d x_{2}, \quad F_{i}^{(n)} \equiv\left[x_{2}^{n} T_{2 i}\right]_{-h}^{h}, \quad \varphi^{(n)} \equiv \int_{-h}^{h} x_{2 \varphi}^{n} d x_{2}, \\
H_{m n} \equiv \int_{-h}^{h} x_{2}^{m} x_{2}^{n} d x_{2}=2 h^{m+n+1} /(m+n+1), \quad m+n \text { even} ; j=0, m+n \text { odd } .
\end{gathered}
$$

Since the variations $\delta u_{i}^{(n)}$ and $\delta \varphi^{(n)}$ are arbitrary, their coefficients in (3.2) and (3.3) must vanish; and we have, as the two dimensional equations of motion and electrostatics of order $n$,

$$
T_{i j, i}^{(n)}-n T_{2 i}^{(n-1)}+F_{i}^{(n)}=\rho \sum_{m=0}^{g} H_{m n} u_{j, t t}^{(m)}, \quad D_{i, i}^{(n)}+(n+1) D_{2}^{(n+1)}=0 .
$$

If $(3.1)_{2}$ is substituted in the three-dimensional equation of electrostatics, $D_{i, i}=0$, the result is

$$
\sum_{n=0}^{0} x_{2}^{n}\left[D_{i, i}^{(n)}+(n+1) D_{2}^{(n+1)}\right]=0,
$$

which is satisfied identically by virtue of $(3.5)_{2}$. This was the motivation for the special form of the expansion of $D_{j}$ in $(3.1)_{2}$.

Substituting $(3.1)_{1}$ in $(2.2)_{1}$ and rearranging terms, we obtain

$$
S_{i j}=\sum_{n=0}^{o} x_{2}^{n} S_{i j}^{(n)}, \quad S_{i j}^{(n)} \equiv \frac{1}{2}\left[u_{i, j}^{(n)}+u_{i, i}^{(n)}+(n+1)\left(\delta_{2 j} u_{i}^{(n+1)}+\delta_{2 i} u_{i}^{(n+1)}\right)\right] .
$$

Next, multiply (2.2) $)_{2}$ by $x_{2}^{n} d x_{2}$ and integrate over the thickness. With (3.4) ${ }_{3}$ and an integration by parts, we find

$$
E_{i}^{(n)} \equiv \int_{-h}^{h} x_{2}^{n} E_{i} d x_{2}=-\varphi_{, i}^{(n)}-\delta_{2 i}\left(\Phi^{(n)}-n \varphi^{(n-1)}\right), \quad \Phi^{(n)} \equiv\left[x_{2}^{n}\right]_{-h}^{h} .
$$

At this stage we have converted (2.1) and (2.2) to two-dimensional form and there remain the constitutive relations (2.3). We substitute $(3.1)_{2}$ and (3.6) in (2.3), multiply by $x_{2}^{m} d x_{2}$ and integrate over the thickness, with the result

$$
T_{i j}^{(m)}=c_{i j k l}^{E} \sum_{n=0}^{g} H_{m n} S_{k l}^{(n)}-e_{k i j} E_{k}^{(m)}, \quad \sum_{n=0}^{o} H_{m n}\left(D_{i}^{(n)}-e_{i k l} S_{k l}^{(n)}\right)=\epsilon_{i k}^{S} E_{k}^{(m)} .
$$


These may be written, in the abbreviated indicial notation, as

$$
T_{p}^{(m)}=c_{p a}^{E} \sum_{n=0}^{o} H_{m n} S_{a}^{(n)}-e_{i p} E_{i}^{(m)}, \quad \sum_{n=0}^{o} H_{m n}\left(D_{i}^{(n)}-e_{i q} S_{a}^{(n)}\right)=\epsilon_{i k}^{S} E_{k}^{(m)},
$$

where $i j$ or $k l=11,22,33,23$ or 32,31 or 13,12 or 21 are replaced by $p$ or $q=1,2,3$, $4,5,6$, respectively; and $S_{p}^{(n)}=S_{i j}^{(n)}, i=j$ and $S_{p}^{(n)}=2 S_{i j}^{(n)}, i \neq j$.

Finally, we define two-dimensional kinetic energy, internal energy and electric enthalpy [3] densities by, respectively,

$$
\begin{gathered}
\mathcal{K} \equiv \int_{-h}^{h} \frac{1}{2} \rho u_{i, t} u_{i, t} d x_{2}=\frac{1}{2} \rho \sum_{m=0}^{g} \sum_{n=0}^{g} H_{m n} u_{i, t}^{(m)} u_{i, t}^{(n)} \\
\mathcal{U} \equiv \int_{-h}^{h} \frac{1}{2}\left(T_{i j} S_{i j}+D_{i} E_{i}\right) d x_{2}=\frac{1}{2} \sum_{n=0}^{g}\left(T_{i j}^{(n)} S_{i j}^{(n)}+E_{i}^{(n)} D_{i}^{(n)}\right)+\frac{1}{2} E_{2}^{(\sigma+1)} D_{2}^{(g+1)}, \\
\mathcal{H C} \equiv \int_{-h}^{h} \frac{1}{2}\left(T_{i j} S_{i j}-D_{i} E_{i}\right) d x_{2}=\frac{1}{2} \sum_{n=0}^{g}\left(T_{i i}^{(n)} S_{i i}^{(n)}-E_{i}^{(n)} D_{i}^{(n)}\right)-\frac{1}{2} E_{2}^{(o+1)} D_{2}^{(o+1)},
\end{gathered}
$$

where $(3.1)_{1},(3.1)_{2},(3.4)_{4}$ and $(3.6)_{1}$ have been used, along with the definitions (3.4) and $(3.7)_{1}$.

4. Truncation of series. In (3.9) we retain only $T_{p}^{(0)}, T_{p}^{(1)}, S_{p}^{(0)} ; S_{p}^{(1)}, D_{i}^{(0)}, D_{i}^{(1)}$, $D_{i}^{(2)}, E_{i}^{(0)}, E_{i}^{(1)}$ and $E_{2}^{(2)}$ whence, tentatively,

$$
\begin{aligned}
& T_{p}^{(0)}=2 h c_{p q}^{E} S_{a}^{(0)}-e_{i p} E_{i}^{(0)}, \quad T_{p}^{(1)}=\frac{2}{3} h^{3} c_{p q}^{E} S_{a}^{(1)}-e_{i p} E_{i}^{(1)}, \\
& 2 h D_{i}^{(0)}+\frac{2}{3} h^{3} D_{i}^{(2)}=2 h e_{i q} S_{a}^{(0)}+\epsilon_{i k}^{S} E_{k}^{(0)},
\end{aligned}
$$

$$
\frac{2}{3} h^{3} D_{i}^{(0)}+\frac{2}{5} h^{5} D_{i}^{(2)}=\frac{2}{3} h^{3} e_{i q} S_{a}^{(0)}+\epsilon_{i 2}^{S} E_{2}^{(2)} .
$$

Eqs. (4.2) may be solved for $D_{i}^{(0)}$ and $D_{i}^{(2)}$ with the result, again tentative,

$$
\begin{aligned}
& D_{i}^{(0)}=e_{i \alpha} S_{a}^{(0)}+(9 / 8 h) \epsilon_{i k}^{S} E_{k}^{(0)}-\left(15 / 8 h^{3}\right) \epsilon_{i 2}^{S} E_{2}^{(2)} \\
& D_{i}^{(2)}=\left(15 / 8 h^{3}\right)\left(3 h^{-2} \epsilon_{i 2}^{S} E_{2}^{(2)}-\epsilon_{i k}^{S} E_{k}^{(0)}\right) .
\end{aligned}
$$

The components $S_{p}^{(0)}$ and $S_{p}^{(1)}$ involve only the displacements $u_{i}^{(0)}, u_{i}^{(1)}$ and $u_{i}^{(2)}$ and we neglect those of higher order. Then, following Cauchy, as in $I$, we neglect the velocity $u_{2, t}^{(1)}$ in the kinetic energy and equations of motion and provide for the free development of the thickness-strain $S_{2}^{(0)}$ by setting $T_{2}^{(0)}=0$ in $(4.1)_{1}$. The condition $T_{2}^{(0)}=0$ permits the elimination of $S_{2}^{(0)}$ from (4.1) ${ }_{1}$ and (4.3) $)_{1}$ with the result, still tentative,

$$
T_{p}^{(0)}=2 h c_{p a}^{*} S_{a}^{(0)}-e_{i p}^{*} E_{i}^{(0)}, \quad D_{i}^{(0)}=e_{i q}^{*} S_{a}^{(0)}+\frac{1}{2} h^{-1} \epsilon_{i j}^{*} E_{j}^{(0)}-\left(15 / 8 h^{3}\right) \epsilon_{i 2}^{S} E_{2}^{(2)},
$$

where

$$
c_{p q}^{*}=c_{p q}^{E}-c_{p 2}^{E} c_{2 q}^{E} / c_{22}^{E}, \quad e_{i p}^{*}=e_{i p}-e_{i 2} c_{p 2}^{E} / c_{22}^{E}, \quad \epsilon_{i j}^{*}=(9 / 4) \epsilon_{i j}^{S}+e_{i 2} e_{j 2} / c_{22}^{E} .
$$

The first order terms are treated as in $I$ : all three velocities $u_{j, t}^{(2)}$ are neglected and free development of the three strains $S_{2 j}^{(1)}$ is accommodated by setting $T_{2 i}^{(1)}=0$ in $(4.1)_{2}$. Then, upon elimination of the $S_{2 i}^{(1)},(4.1)_{2}$ and $(4.1)_{3}$ become

$$
T_{r}^{(1)}=\frac{2}{3} h^{3} \gamma_{r s} S_{s}^{(1)}-\psi_{i r} E_{i}^{(1)}, \quad D_{i}^{(1)}=\psi_{i r} S_{r}^{(1)}+\frac{3}{2} h^{-3} \zeta_{i j} E_{i}^{(1)},
$$

where, with $i, j=1,2,3 ; r, s=1,3,5 ; v, w=2,4,6$ :

$$
\gamma_{r s}=c_{r s}^{E}-c_{r w}^{E} c_{v s}^{E}\left(c_{v w}^{E}\right)^{-1}, \quad \psi_{i r}=e_{i r}-e_{i v} c_{r w}^{E}\left(c_{v w}^{E}\right)^{-1}, \quad \zeta_{i j}=\epsilon_{i j}^{S}+e_{i v} e_{j w}\left(c_{v w}^{E}\right)^{-1} .
$$


At this stage, the electric enthalpy is

$$
\begin{aligned}
\mathfrak{F}= & \frac{1}{2}\left(T_{p}^{(0)} S_{p}^{(0)}+T_{p}^{(1)} S_{p}^{(1)}-E_{i}^{(0)} D_{i}^{(0)}-E_{i}^{(1)} D_{i}^{(1)}-E_{2}^{(2)} D_{2}^{(2)}\right) \\
= & \frac{1}{2}\left[2 h c_{p a}^{*} S_{p}^{(0)} S_{a}^{(0)}-2 e_{i p}^{*} S_{p}^{(0)} E_{i}^{(0)}+\frac{2}{3} h^{3} \gamma_{r s} S_{r}^{(1)} S_{s}^{(1)}-2 \psi_{i r} E_{i}^{(1)} S_{r}^{(1)}-\frac{1}{2} h^{-1} \epsilon_{i j}^{*} E_{i}^{(0)} E_{j}^{(0)}\right. \\
& \left.\quad-\frac{3}{2} h^{-3} \zeta_{i j} E_{i}^{(1)} E_{i}^{(1)}+(15 / 4) h^{-3} \epsilon_{2 k}^{S} E_{2}^{(2)} E_{k}^{(0)}-(45 / 8) h^{-5} \epsilon_{22}^{S} E_{2}^{(2)} E_{2}^{(2)}\right],
\end{aligned}
$$

and an analogous expression may be written for the internal energy $\mathcal{u}$.

The final step in the process of truncation is to introduce the usual shear-correction factors by replacing $S_{4}^{(0)}$ and $S_{6}^{(0)}$, in $\mathfrak{H C}$ and $\mathcal{U}$, with $\kappa_{4} S_{4}^{(0)}$ and $\kappa_{6} S_{6}^{(0)}$, where the values of $\kappa_{4}$ and $\kappa_{6}$ are to be fixed so as to adjust the two thickness-shear frequencies to their exact values. The correction factors may be inserted in the appropriate positions by replacing $c_{p e}^{*}$ and $e_{i p}^{*}$, in $\mathcal{F C}$ and $\mathcal{U}$, by

$$
c_{p a}^{* *}=\kappa_{p}^{\alpha} \kappa_{a}^{\beta} c_{p a}^{*}, \quad e_{i a}^{* *}=\kappa_{a}^{\beta} e_{i q}^{*} \quad \text { (no sum) }
$$

where $\alpha$ and $\beta$ are the powers $\alpha=\cos ^{2}(p \pi / 2), \beta=\cos ^{2}(q \pi / 2)$. Thus, $\kappa_{p}^{\alpha}\left(\right.$ or $\left.\kappa_{q}^{\beta}\right)$ is equal to $\kappa_{4}, \kappa_{6}$ or 1 according as $p$ (or $q$ ) in $c_{p a}^{*}$ and $e_{i p}^{*}$ is 4,6 or neither, respectively. The same definitions hold for $c_{i j k l}^{* *}, e_{i k l}^{* *}, \kappa_{i j}^{\alpha}, \kappa_{k l}^{\beta}$ if $p$ and $q$ are replaced by $i j$ and $k l$.

To recapitulate, after the truncation and adjustments, we have the following*:

Electric enthalpy density: $\mathfrak{F C}$ is given by (4.8) with $c_{p q}^{*}$ and $e_{i p}^{*}$ replaced by $c_{p q}^{* *}$ and $e_{i p}^{* *}$. Internal energy density:

$$
\mathcal{U}=\mathfrak{F C}+E_{i}^{(0)} D_{i}^{(0)}+E_{i}^{(1)} D_{i}^{(1)}+E_{2}^{(2)} D_{2}^{(2)}
$$

Kinetic energy density:

$$
\Re=\rho h\left(u_{i, \ell}^{(0)} u_{i, t}^{(0)}+\frac{1}{3} h^{2} u_{a, \ell}^{(1)} u_{a, t}^{(1)}\right) .
$$

Constitutive relations:

$$
\begin{aligned}
& T_{p}^{(0)}=\partial \mathcal{F C} / \partial S_{p}^{(0)}=2 h c_{p a}^{* *} S_{a}^{(0)}-e_{k p}^{* *} E_{k}^{(0)} \\
& T_{r}^{(1)}=\partial \mathcal{H C} / \partial S_{r}^{(1)}=\frac{2}{3} h^{3} \gamma_{r s} S_{s}^{(1)}-\psi_{i r} E_{i}^{(1)}, \\
& D_{i}^{(0)}=-\partial \mathcal{H C} / \partial E_{i}^{(0)}=e_{i a}^{* *} S_{a}^{(0)}+\frac{1}{2} h^{-1} \epsilon_{i j}^{*} E_{i}^{(0)}-\left(15 / 8 h^{3}\right) \epsilon_{i 2}^{S} E_{2}^{(2)}, \\
& D_{i}^{(1)}=-\partial \mathcal{H C} / \partial E_{i}^{(1)}=\psi_{i r} S_{r}^{(1)}+\frac{3}{2} h^{-3} \zeta_{i j} E_{i}^{(1)}, \\
& D_{2}^{(2)}=-\partial \mathcal{H C} / \partial E_{2}^{(2)}=\left(15 / 8 h^{5}\right)\left(3 \epsilon_{22}^{S} E_{2}^{(2)}-h^{2} \epsilon_{2 k}^{S} E_{k}^{(0)}\right) .
\end{aligned}
$$

Equations of motion:

$$
T_{i j, i}^{(0)}+F_{i}^{(0)}=2 \rho h u_{i, t t}^{(0)}, \quad T_{a b, a}^{(1)}-T_{2 b}^{(0)}+F_{b}^{(1)}=\frac{2}{3} \rho h^{3} u_{b, t \ell}^{(1)} .
$$

Equations of electrostatics:

$$
D_{i, i}^{(0)}+D_{2}^{(1)}=0, \quad D_{i, i}^{(1)}+2 D_{2}^{(2)}=0
$$

Strain-displacement relations:

$$
S_{i j}^{(0)}=\frac{1}{2}\left(u_{i, j}^{(0)}+u_{i, i}^{(0)}+\delta_{2 j} u_{i}^{(1)}+\delta_{2 i} u_{i}^{(1)}\right), \quad S_{a b}^{(1)}=\frac{1}{2}\left(u_{a, b}^{(1)}+u_{b, a}^{(1)}\right) .
$$

${ }^{*}$ Here and in the sequel indices $a, b, c, d$ range over 1,$3 ; r, s$ over $1,3,5 ; i, j, k, l$ over $1,2,3$. 
Electric field-potential relations:

$E_{i}^{(0)}=-\varphi_{, i}^{(0)}-\delta_{i 2} \Phi^{(0)}, E_{i}^{(1)}=-\varphi_{, i}^{(1)}+\delta_{i 2}\left(\varphi^{(0)}-\Phi^{(1)}\right), E_{2}^{(2)}=2 \varphi^{(1)}-\Phi^{(2)}$,

and we note that $\Phi^{(2)}=h^{2} \Phi^{(0)}$.

Eqs. (4.12)-(4.16) comprise thirty-seven equations in the thirty-seven variables: five each of $T_{p}^{(0)}$ and $S_{p}^{(0)}$; three each of $T_{r}^{(1)}, S_{r}^{(1)}, D_{i}^{(0)}, E_{i}^{(0)}, D_{i}^{(1)}, E_{i}^{(1)}$ and $u_{i}^{(0)}$; two $u_{a}^{(1)}$; and $D_{2}^{(2)}, E_{2}^{(2)}, \varphi^{(0)}$ and $\varphi^{(1)}$. The thirty-seven equations may be reduced to seven in seven variables by first substituting (4.15) and (4.16) in (4.12), with the result

$$
\begin{aligned}
& T_{i j}^{(0)}=2 h c_{i j k l}^{* *}\left(u_{k, l}^{(0)}+\delta_{2 l} u_{k}^{(1)}\right)+e_{k i j}^{* *} \varphi_{, k}^{(0)}+e_{2 i j}^{* *} \Phi^{(0)}, \\
& T_{a b}^{(1)}=\frac{2}{3} h^{3} \gamma_{a b c d} u_{c, d}^{(1)}-\psi_{2 a b} \varphi^{(0)}+\psi_{i a b} \varphi_{, i}^{(1)}+\psi_{2 a b} \Phi^{(1)}, \\
& D_{i}^{(0)}=e_{i k l}^{* *}\left(u_{k, l}^{(0)}+\delta_{2 l} u_{k}^{(1)}\right)-\frac{1}{2} h^{-1} \epsilon_{i j}^{*} \varphi_{, j}^{(0)}-\left(15 / 4 h^{3}\right) \epsilon_{i 2}^{S} \varphi^{(1)}-\frac{1}{8} h^{-1} \epsilon_{i 2}^{\prime} \Phi^{(0)}, \\
& D_{i}^{(1)}=\psi_{i a b} u_{a, b}^{(1)}+\frac{3}{2} h^{-3} \zeta_{i 2} \varphi^{(0)}-\frac{3}{2} h^{-3} \zeta_{i j} \varphi_{, i}^{(1)}-\frac{3}{2} h^{-3} \zeta_{i 2} \Phi^{(1)}, \\
& D_{2}^{(2)}=\left(15 / 8 h^{5}\right)\left(h^{2} \epsilon_{2 k}^{S} \varphi_{, k}^{(0)}+6 \epsilon_{22}^{S} \varphi^{(1)}-2 h_{\epsilon_{22}}^{2} \Phi^{(0)}\right),
\end{aligned}
$$

where $\epsilon_{i 2}^{\prime}=4 \epsilon_{i 2}^{*}-15 \epsilon_{i 2}^{S}$; and then substituting (4.17) into (4.13) and (4.14) to obtain

$$
\begin{gathered}
2 h c_{i j k l}^{* *}\left(u_{k, l i}^{(0)}+\delta_{2 k} u_{l, i}^{(1)}\right)+e_{k i j}^{* *} \varphi_{, k i}^{(0)}+F_{j}^{(0)}+e_{2 i j}^{* *} \Phi_{, i}^{(0)}=2 \rho h u_{j, t t}^{(0)}, \\
\frac{2}{3} h^{3} \gamma_{a b c d} u_{c, d a}^{(1)}-2 h c_{2 b k l}^{* *}\left(u_{k, l}^{(0)}+\delta_{2 k} u_{l}^{(1)}\right)-e_{i 2 b}^{* *} \varphi_{, i}^{(0)}+\psi_{i a b}\left(\varphi_{, i a}^{(1)}-\delta_{2 i} \varphi_{, a}^{(0)}\right) \\
+F_{b}^{(1)}-e_{22 b}^{* *} \Phi^{(0)}+\psi_{2 a b} \Phi_{, a}^{(1)}=\frac{2}{3} \rho h^{3} u_{b, t t}^{(1)}, \\
\frac{2}{3} h^{3} \psi_{2 k l} u_{k, l}^{(1)}+\frac{2}{3} h^{3} e_{i k l}^{* *}\left(u_{k, l i}^{(0)}+\delta_{2 k} u_{l, i}^{(1)}\right)-\frac{1}{3} h^{2} \epsilon_{i j}^{* *} \varphi_{, i j}^{(0)}-\zeta_{2 i}\left(\varphi_{, j}^{(1)}-\delta_{2 j} \varphi^{(0)}\right) \\
-5 / 2 \epsilon_{k 2}^{S} \varphi_{, k}^{(1)}-(1 / 12) h^{2} \epsilon_{i 2}^{\prime} \Phi_{, i}^{(0)}-\zeta_{22} \Phi^{(1)}=0, \\
\frac{2}{3} h^{3} \psi_{i a b} u_{a, b i}^{(1)}-\zeta_{i j}\left(\varphi_{, i j}^{(1)}-\delta_{2 i} \varphi_{, i}^{(0)}\right)+5 / 2 \epsilon_{2 k}^{S} \varphi_{, k}^{(0)}+15 h^{-2} \epsilon_{22}^{S} \varphi^{(1)} \\
-5 \epsilon_{22}^{S} \Phi^{(0)}-\zeta_{i 2} \Phi_{, i}^{(1)}=0 .
\end{gathered}
$$

These are seven second order differential equations in the seven dependent variables $u_{i}^{(0)}, u_{a}^{(1)}, \varphi^{(0)}$ and $\varphi^{(1)}$ with seven non-homogeneous (forcing) terms: the five surface tractions $F_{i}^{(0)}, F_{b}^{(1)}$ and the two surface potentials $\Phi^{(0)}, \Phi^{(1)}$.

5. Uniqueness of solution. To establish a theorem of uniqueness of solution of the thirty-seven equations (4.12)-(4.16)' we consider two sets of the thirty-seven variables, each set satisfying the equations, and form a system comprising the thirty-seven differences between corresponding variables in the two sets. In terms of variables in the difference-system, we form the equation

$\int_{t_{0}}^{t} d t \int_{A}\left[\left(T_{i j, i}^{(0)}+F_{i}^{(0)}-2 \rho h u_{j, t t}^{(0)}\right) u_{i, t}^{(0)}+\left(T_{a b, a}^{(1)}-T_{2 b}^{(0)}+F_{b}^{(1)}-\frac{2}{3} \rho h^{3} u_{b, t t}^{(1)}\right) u_{b, t}^{(1)}\right] d A=0$,

which can be transformed, by a process analogous to that employed in $I$, to

$$
\begin{aligned}
\int_{A}[u & +\mathcal{K}]_{t_{0}}^{t} d A=\int_{t_{0}}^{t} d t \oint_{\mathrm{C}} n_{a}\left(T_{a j}^{(0)} u_{j, t}^{(0)}+T_{a b}^{(1)} u_{b, t}^{(1)}-\varphi^{(0)} D_{a, t}^{(0)}-\varphi^{(1)} D_{a, t}^{(1)}\right) d s \\
& +\int_{t_{0}}^{t} d t \int_{A}\left[F_{j}^{(0)} u_{j_{,}}^{(0)}+F_{b}^{(1)} u_{b, t}^{(1)}-\Phi^{(0)}\left(D_{2, t}^{(0)}+h^{2} D_{2, t}^{(2)}\right)-\Phi^{(1)} D_{2, t}^{(1)}\right] d A,
\end{aligned}
$$

in which the line integral is around the edge, $C$, of the plate and the $n_{a}$ are the components 
of the outward normal in the plane of the plate. From (5.1), by the usual argument, there are seven conditions to be specified at each point of the interior and at each point on the edge of the plate in addition to the initial values of $u_{i}^{(0)}, u_{a}^{(1)}, \varphi^{(0)}, \varphi^{(1)}, u_{i, t}^{(0)}$ and $u_{a, t}^{(1)}$. Referred to orthogonal coordinates $n, s, x_{2}$, the edge conditions are one member of each of the seven products

$$
T_{n n}^{(0)} u_{n}^{(0)}, \quad T_{n s}^{(0)} u_{s}^{(0)}, \quad T_{n 2}^{(0)} u_{2}^{(0)}, \quad T_{n n}^{(1)} u_{n}^{(1)}, \quad T_{n s}^{(1)} u_{s}^{(1)}, \quad \varphi^{(0)} D_{n}^{(0)}, \quad \varphi^{(1)} D_{n}^{(1)} .
$$

In terms of orthogonal coordinates $\alpha, \beta, x_{2}$, the interior conditions are one member of each of the seven products

$$
F_{\alpha}^{(0)} u_{\alpha}^{(0)}, \quad F_{\beta}^{(0)} u_{\beta}^{(0)}, \quad F_{2}^{(0)} u_{2}^{(0)}, \quad F_{\alpha}^{(1)} u_{\alpha}^{(1)}, \quad F_{\beta}^{(1)} u_{\beta}^{(1)}, \quad \Phi^{(0)}\left(D_{2}^{(0)}+h^{2} D_{2}^{(2)}\right), \quad \Phi^{(1)} D_{2}^{(1)} .
$$

The terms $D_{2}^{(0)}+h^{2} D_{2}^{(2)}$ and $D_{2}^{(1)}$, in the interior conditions, can be interpreted by recalling that, to our approximation,

Hence

$$
D_{2}=D_{2}^{(0)}+x_{2} D_{2}^{(1)}+x_{2}^{2} D_{2}^{(2)} \text {. }
$$

$$
D_{2}^{(0)}+h^{2} D_{2}^{(2)}=\frac{1}{2}\left[D_{2}(h)+D_{2}(-h)\right] \text { and } D_{2}^{(1)}=\frac{1}{2} h^{-1}\left[D_{2}(h)-D_{2}(-h)\right] .
$$

6. Orthogonal functions. Consider two solutions

$$
\begin{aligned}
& \left(u_{i}^{(0)}, u_{b}^{(1)}, \varphi^{(0)}, \varphi^{(1)}\right)=\left(u_{i}^{(0) \mu}, u_{b}^{(1) \mu}, \varphi^{(0) \mu}, \varphi^{(1) \mu}\right) e^{i \omega t}, \\
& \left(u_{j}^{(0)}, u_{b}^{(1)}, \varphi^{(0)}, \varphi^{(1)}\right)=\left(u_{j}^{(0) \nu}, u_{b}^{(1) \nu}, \varphi^{(0) \nu}, \varphi^{(1) \nu}\right) e^{i \omega \nu t},
\end{aligned}
$$

of the equations of motion (4.13) and electrostatics (4.14) with $\left(F_{i}^{(0)}, F_{b}^{(1)}\right)=0$. By a process similar to that employed in $I$, we find

$$
\begin{aligned}
& 2 \rho h\left(\omega_{\mu}^{2}-\omega_{\nu}^{2}\right) \int_{\Delta}\left(u_{i}^{(0) \mu} u_{i}^{(0) \nu}+\frac{1}{3} h^{2} u_{b}^{(1) \mu} u_{b}^{(1) \nu}\right) d A \\
& \quad=\int_{A}\left[\Phi^{(0) \mu}\left(D_{2}^{(0) \nu}+h^{2} D_{2}^{(2) \nu}\right)-\Phi^{(0) \nu}\left(D_{2}^{(0) \mu}+h^{2} D_{2}^{(2) \mu}\right)+\Phi^{(1) \mu} D_{2}^{(1) \nu}-\Phi^{(1) \nu} D_{2}^{(1) \mu}\right] d A \\
& \quad+\oint_{c} n_{a}\left(T_{a i}^{(0) \nu} u_{i}^{(0) \mu}-T_{a i}^{(0) \mu} u_{i}^{(0) \nu}+T_{a b}^{(1) \nu} u_{b}^{(1) \mu}-T_{a b}^{(1) \mu} u_{b}^{(1) \nu}\right) d s \\
& \quad+\oint_{c} n_{a}\left(D_{a}^{(0) \nu} \varphi^{(0) \mu}-D_{a}^{(0) \mu} \varphi^{(0) \nu}+D_{a}^{(1) \nu} \varphi^{(1) \mu}-D_{a}^{(1) \mu} \varphi^{(1) \nu}\right) d s .
\end{aligned}
$$

The surface integral in (6.1) vanishes for either zero electric potential or zero electric displacement on both faces of the plate; and the line integrals vanish for homogeneous boundary conditions on the edge. Thus, for homogeneous conditions on the faces and edge, (6.1) becomes

$$
\int_{\Lambda}\left(u_{i}^{(0) \mu} u_{j}^{(0) \nu}+\frac{1}{3} h^{2} u_{b}^{(1) \mu} u_{b}^{(1) \nu}\right) d A=N_{(\mu)} \delta_{\mu \nu},
$$

where $N_{(\mu)}$ is a normalization factor and $\delta_{\mu \nu}$ is the Kronecker delta.

7. Steady forced vibrations. A problem of technological interest is that of steady vibrations forced by an ac-voltage applied to electrodes on the faces of a plate with its edge free. This case is included in solutions of (4.18) with prescribed tractions $F_{i}^{(0)}, F_{b}^{(1)}$ and potentials $\Phi^{(0)}, \Phi^{(1)}$ on the faces and with boundary conditions

$$
n_{a} T_{a i}^{(0)}=n_{a} T_{a b}^{(1)}=n_{a} D_{a}^{(0)}=n_{a} D_{a}^{(1)}=0
$$


on the edge. Although (7.1) appear to be homogeneous boundary conditions, they are actually inhomogeneous due to the presence of the prescribed potentials $\Phi^{(0)}$ and $\Phi^{(1)}$ in (4.17). A general solution will be obtained by an adaptation of the classical device [4] of introducing an auxiliary function which transfers the boundary inhomogeneity to the differential equations, leaving a residual problem of inhomogeneous differential equations and homogeneous boundary conditions. In our case, the eqautions of motion, $(4.18)_{1}$ and $(4.18)_{2}$, and electrostatics, $(4.18)_{3}$ and $(4.18)_{4}$, are initially inhomogeneous. We shall choose the auxiliary function so that it removes the inhomogeneity not only from the boundary conditions but also from the electrostatic equations, leaving a residuai problem of inhomogeneous equations of motion, homogeneous electrostatic equations and homogeneous boundary conditions. Let

$\left(u_{i}^{(0)}, u_{b}^{(1)}, \varphi^{(0)}, \varphi^{(1)}\right)=\left(u_{i}^{(0) A}, u_{b}^{(1) A}, \varphi^{(0) A}, \varphi^{(1) A}\right) e^{i \omega t}+\left(u_{i}^{(0) R}, u_{b}^{(1) R}, \varphi^{(0) R}, \varphi^{(1) R}\right) e^{i \omega t}$,

where the superscript $A$ identifies the auxiliary functions and $R$ the residual solution. Inserting (7.2) in (4.17) and cancelling the factor $e^{i \omega t}$, we obtain expressions of the type

$$
T_{i j}^{(0)}=T_{i j}^{(0) R}+T_{i j}^{(0) A}+e_{2 i i}^{* *} \Phi^{(0)},
$$

where

$$
\begin{aligned}
& T_{i j}^{(0) R}=2 h c_{i j k l}^{* *}\left(u_{k, l}^{(0) R}+\delta_{2 l} u_{k}^{(1) R}\right)+e_{k i j}^{* *} \varphi_{, k}^{(0) R}, \\
& T_{i j}^{(0) A}=2 h c_{i j k l}^{* *}\left(u_{k, l}^{(0) A}+\delta_{2 l} u_{k}^{(1) A}\right)+e_{k i j}^{* *} \varphi_{, k}^{(0) A} .
\end{aligned}
$$

With resolutions like (7.3) for all of (4.17), Eqs. (4.8) become

$$
\begin{gathered}
T_{i i, i}^{(0) R}+2 \rho h \omega^{2} u_{i}^{(0) R}+T_{i j, i}^{(0) A}+2 \rho h \omega^{2} u_{i}^{(0) A}+F_{i}^{(0)}+e_{2 i j}^{* *} \Phi_{, i}^{(0)}=0, \\
T_{a b, a}^{(1) R}-T_{2 b}^{(0) R}+\frac{2}{3} \rho h^{3} \omega^{2} u_{b}^{(1) R}+T_{a b, a}^{(1) A}-T_{2 b}^{(0) A} \\
+\frac{2}{3} \rho h^{3} \omega^{2} u_{b}^{(1) A}+F_{b}^{(1)}-e_{22 b}^{* *} \Phi^{(0)}+\psi_{2 a b} \Phi_{, a}^{(1)}=0 \\
D_{i, i}^{(0) R}+D_{2}^{(1) R}+D_{i, i}^{(0) A}+D_{2}^{(1) A}-\frac{1}{8} h^{-1} \epsilon_{i 2}^{\prime} \Phi_{, i}^{(0)}-\frac{3}{2} h^{-3} \zeta_{22} \Phi^{(1)}=0 \\
D_{i, i}^{(1) R}+2 D_{2}^{(2) R}+D_{i, i}^{(1) A}+2 D_{2}^{(2) A}-\left(15 / 2 h^{3}\right) \epsilon_{22}^{S} \Phi^{(0)}-\frac{3}{2} h^{-3} \zeta_{i 2} \Phi_{, i}^{(1)}=0,
\end{gathered}
$$

and the boundary conditions (7.1) become

$$
\begin{aligned}
& n_{a} T_{a i}^{(0) R}+n_{a} T_{a i}^{(0) A}+n_{a} e_{2 a i}^{* *} \Phi^{(0)}=0, n_{a} T_{a b}^{(1) R}+n_{a} T_{a b}^{(1) A}+n_{a} \psi_{2 a b} \Phi^{(1)}=0, \\
& n_{a} D_{a}^{(0) R}+n_{a} D_{a}^{(0) A}-\frac{1}{8} h^{-1} n_{a} \epsilon_{a 2}^{\prime} \Phi^{(0)}=0, n_{a} D_{a}^{(1) R}+n_{a} D_{a}^{(1) A}-\frac{3}{2} h^{-3} n_{a} \zeta_{a 2} \Phi^{(1)}=0 .
\end{aligned}
$$

We now choose the auxiliary functions $u_{i}^{(0) A}, u_{a}^{(1) A}, \varphi^{(0) A}, \varphi^{(1) A}$ to be particular solutions of the differential equations

$$
\begin{aligned}
D_{i, i}^{(0) A}+D_{2}^{(1) A}=\frac{1}{8} h^{-1} \epsilon_{i 2}^{\prime} \Phi_{, i}^{(0)}+\frac{3}{2} h^{-3} \zeta_{22} \Phi^{(1)}, \\
D_{i, i}^{(1) A}+2 D_{2}^{(2) A}=(15 / 2) h^{-3} \epsilon_{22}^{S} \Phi^{(0)}+\frac{3}{2} h^{-3} \zeta_{i 2} \Phi_{, i}^{(1)},
\end{aligned}
$$

and to satisfy the boundary conditions

$$
\begin{aligned}
& n_{a} T_{a i}^{(0) A}+n_{a} e_{2 a j}^{* *} \Phi^{(0)}=0, \quad n_{a} T_{a b}^{(1) A}+n_{a} \psi_{2 a b} \Phi^{(1)}=0, \\
& n_{a} D_{a}^{(0) A}-\frac{1}{8} h^{-1} n_{a} \epsilon_{a 2}^{\prime} \Phi^{(0)}=0, \quad n_{a} D_{(a)}^{(1) A}-\frac{3}{2} h^{-3} n_{a} \zeta_{a 2} \Phi^{(1)}=0 .
\end{aligned}
$$

Then, inserting (7.6) and (7.7) in (7.4) and (7.5), respectively, we find the residual 
problem governed by the differential equations

$$
\begin{gathered}
T_{i, i}^{(0) R}+2 \rho h \omega^{2} u_{i}^{(0) R}+2 \rho h \omega^{2} u_{j}^{(0) A}+G_{j}^{(0)}=0, \\
T_{a b, a}^{(1) R}-T_{2 b}^{(0) R}+\frac{2}{3} \rho h^{3} \omega^{2} u_{b}^{(1) R}+\frac{2}{3} \rho h^{3} \omega^{2} u_{b}^{(1) A}+\frac{1}{3} h^{2} G_{b}^{(1)}=0, \\
D_{i, i}^{(0) R}+D_{2}^{(1) R}=0, \quad D_{i, i}^{(1) R}+2 D_{2}^{(2) R}=0,
\end{gathered}
$$

and the boundary conditions

$$
n_{a} T_{a j}^{(0) R}=n_{a} T_{a b}^{(1) R}=n_{a} D_{a}^{(0) R}=n_{a} D_{a}^{(1) R}=0,
$$

where

$$
\begin{gathered}
G_{j}^{(0)}=T_{i j, i}^{(0) A}+F_{i}^{(0)}+e_{2 i j}^{* *} \Phi_{, i}^{(0)}, \\
G_{b}^{(1)}=3 h^{-2}\left(T_{a b, a}^{(1) A}-T_{2 b}^{(0) A}+F_{b}^{(1)}-e_{22 b}^{* *} \Phi^{(0)}+\psi_{2 a b} \Phi_{, a}^{(1)}\right) .
\end{gathered}
$$

The solution of the residual problem may be expressed as an infinite series

$$
\left(u_{i}^{(0) R}, u_{b}^{(1) R}, \varphi^{(0) R}, \varphi^{(1) R}\right)=\sum_{\mu} A_{\mu}\left(u_{j}^{(0) \mu}, u_{b}^{(1) \mu}, \varphi^{(0) \mu}, \varphi^{(1) \mu}\right),
$$

where $u_{i}^{(0) \mu}, u_{b}^{(1) \mu}, \varphi^{(0) \mu}, \varphi^{(1) \mu}$ are the orthogonal solutions of the homogeneous $\left(F_{i}^{(0)}=\right.$ $F_{b}^{(1)}=\Phi^{(0)}=\Phi^{(1)}=0$ ) system

$$
\begin{gathered}
T_{i j, i}^{(0) \mu}+2 \rho h \omega_{\mu}^{2} u_{i}^{(0) \mu}=0, \quad T_{a b, a}^{(1) \mu}-T_{2 b}^{(0) \mu}+\frac{2}{3} \rho h^{3} \omega_{\mu}^{2} u_{b}^{(1) \mu}=0, \\
D_{i, i}^{(0) \mu}+D_{2}^{(1) \mu}=0, \quad D_{i, i}^{(1) \mu}+2 D_{2}^{(2) \mu}=0, \\
n_{a} T_{a i}^{(0) \mu}=n_{a} T_{a b}^{(1) \mu}=n_{a} D_{a}^{(0) \mu}=n_{a} D_{a}^{(1) \mu}=0 .
\end{gathered}
$$

Noting that, from (7.11) and (4.17), with $\Phi^{(0)}=\Phi^{(1)}=0$,

$$
\left(T_{i j}^{(0) R}, T_{a b}^{(1) R}, D_{i}^{(0) R}, D_{i}^{(1) R}, D_{2}^{(2) R}\right)=\sum_{\mu} A_{\mu}\left(T_{i j}^{(0) \mu}, T_{a b}^{(1) \mu}, D_{i}^{(0) \mu}, D_{i}^{(1) \mu}, D_{2}^{(2) \mu}\right),
$$

we see, from (7.13), that the electrostatic equations (7.10) are satisfied identically by the solution (7.11). To find the $A_{\mu}$ which will make (7.11) satisfy the equation of motions (7.8) and (7.9), we must first express the known inhomogeneous terms; in the latter, in series form:

$$
\begin{array}{rlrl}
G_{i}^{(0)} & =\sum_{\mu} B_{\mu} u_{i}^{(0) \mu}, & u_{i}^{(0) A}=\sum_{\mu} C_{\mu} u_{i}^{(0) \mu}, \\
G_{b}^{(1)} & =\sum_{\mu} B_{\mu} u_{b}^{(1) \mu}, & u_{b}^{(1) A} & =\sum_{\mu} C_{\mu} u_{b}^{(1) \mu} .
\end{array}
$$

Multiplying (7.16) by $u_{i}^{(0) \nu},(7.17)$ by $\frac{1}{3} h^{2} u_{b}^{(1) \nu}$, adding, integrating over the area of the plate and using (6.2), we find

$$
\begin{aligned}
& B_{\mu}=N_{(\mu)}^{-1} \int_{A}\left(G_{i}^{(0)} u_{j}^{(0) \mu}+\frac{1}{3} h^{2} G_{b}^{(1)} u_{b}^{(1) \mu}\right) d A \\
& C_{\mu}=N_{(\mu)}^{-1} \int_{A}\left(u_{i}^{(0) A} u_{j}^{(0) \mu}+\frac{1}{3} h^{2} u_{b}^{(1) A} u_{b}^{(1) \mu}\right) d A .
\end{aligned}
$$

Then, substituting (7.11), (7.15), (7.16) and (7.17) in (7.8) and (7.9), using (7.12), multiplying (7.8) by $u_{j}^{(0) v}$ and (7.9) by $u_{b}^{(1) v}$, adding, integrating over the area and then using (6.2), we find

$$
A_{\mu}=\left(B_{\mu}+2 \rho h \omega^{2} C_{\mu}\right) / 2 \rho h\left(\omega_{\mu}^{2}-\omega^{2}\right)
$$


Thus we have

$$
\begin{aligned}
\left(u_{i}^{(0)}, u_{b}^{(1)}, \varphi^{(0)}, \varphi^{(1)}\right)=\left(u_{i}^{(0) A}, u_{b}^{(1) A}, \varphi^{(0) A},\right. & \left.\varphi^{(1) A}\right) e^{i \omega t} \\
& +\sum_{\mu} A_{\mu}\left(u_{i}^{(0) \mu}, u_{b}^{(1) \mu}, \varphi^{(0) \mu}, \varphi^{(1) \mu}\right) e^{i \omega t}
\end{aligned}
$$

as the complete, steady state solution of (4.18) with boundary conditions (7.1).

8. Surface charge. If the ac-voltage is applied uniformly over traction-free faces, by means of a thin, perfectly conducting film, we have

$$
F_{i}^{(0)}=F_{b}^{(1)}=\Phi^{(1)}=0, \quad \Phi^{(0)}=V e^{i \omega t},
$$

where $V$ is the constant voltage drop across the thickness. The current through the plate is proportional to the integrated surface charge, $Q$, over the whole area of a face, where

$$
\left.Q=\int_{A} D_{2}\right]_{x_{2}=-h} d A .
$$

Inserting (5.2) in (8.2) and using (4.14), the divergence theorem and boundary conditions $(7.1)$, we find

$$
Q=\int_{A} D_{2}^{(0)} d A .
$$

Then, substituting (7.20) in the third of (4.17) and the resulting expression for $D_{2}^{(0)}$ in (8.3) we have, for the total surface charge,

$$
Q=V\left(L-\frac{1}{8} h^{-1} \epsilon_{22}^{\prime} A+\sum_{\mu} A_{\mu} Y_{\mu}\right),
$$

where $A$ is the area of the plate, $A_{\mu}$ is given by (7.19) and

$$
\begin{aligned}
& Y_{\mu}=\int_{A}\left[e_{2 k l}^{* *}\left(u_{k, l}^{(0) \mu}+\delta_{2 l} u_{k}^{(1) \mu}\right)-\frac{1}{2} h^{-1} \epsilon_{2 j}^{*} \varphi_{, i}^{(1) \mu}-\left(15 / 4 h^{3}\right) \epsilon_{22}^{S} \varphi^{(1) \mu}\right] d A, \\
& L=\int_{A}\left[e_{2 k l}^{* *}\left(u_{k, l}^{(0) A}+\delta_{2 l} u_{k}^{(1) A}\right)-\frac{1}{2} h^{-1} \epsilon_{2 j}^{*} \varphi_{, i}^{(1) A}-\left(15 / 4 h^{3}\right) \epsilon_{22}^{S} \varphi^{(1) A}\right] d A,
\end{aligned}
$$

with the constant $V$ factored out. The formula (8.4), for surface charge, takes account of the action of the crystal both as a capacitor, in the terms outside the summation sign, and as a charge generator; including, in both cases, the distortion of the field due to the finite dimensions of the plate.

9. Application to rectangular, rotated- $Y$-cut, quartz plates. Quartz is a trigonal crystal and a rotated- $Y$-cut is a plate which contains a digonal axis and whose normal makes an angle, say $\theta$, with the trigonal axis [3]. When referred to rectangular axes in and normal to the plane of the plate, the constitutive relations exhibit monoclinic symmetry. We take $x_{1}$ as the digonal axis in the plane of the plate. A solution [5] of the equations in $I$ for modes independent of $x_{3}$ has given a frequency spectrum of coupled thickness-shear, flexure and face-shear modes, for rectangular $A T$-cut plates $(\theta=$ $35^{\circ} 15^{\prime}$ ), very close to that determined experimentally by Koga and Fukuyo [6]. We shall obtain the analogous solution of the present equations for the general rotated- $Y$-cut and give the formula for the integrated surface charge. 
With (8.1), monoclinic symmetry and independence of $x_{3}$, we may separate, from (4.18), the four equations

$$
\begin{gathered}
c_{66} \kappa_{6}^{2}\left(u_{2,11}^{(0)}+u_{1,1}^{(1)}\right)+c_{56} \kappa_{6} u_{3,11}^{(0)}+\rho \omega^{2} u_{2}^{(0)}=0, \\
c_{56} \kappa_{6}\left(u_{2,11}^{(0)}+u_{1,1}^{(1)}\right)+c_{55} u_{3,11}^{(0)}+\rho \omega^{2} u_{3}^{(0)}=0, \\
\frac{2}{3} h^{3} Y_{11} u_{1,11}^{(1)}-2 h \kappa_{6}^{2} c_{66}\left(u_{2,1}^{(0)}+u_{1}^{(1)}\right)-2 h \kappa_{6} c_{56} u_{3,1}^{(0)} \\
+2 h \psi_{11} \varphi_{11}^{(1)}+\frac{2}{3} h^{3} \rho \omega^{2} u_{1}^{(1)}-\kappa_{6} e_{26} V=0, \\
\frac{2}{3} h^{3} \psi_{11} u_{1,11}^{(1)}-\zeta_{11} \varphi_{, 11}^{(1)}+15 h^{-2} \epsilon_{22}^{S} \varphi^{(1)}-5 \epsilon_{22}^{S} V=0,
\end{gathered}
$$

in the four variables $u_{2}^{(0)}, u_{3}^{(0)}, u_{1}^{(1)}$ and $\varphi_{i}^{(1)}$ while $u_{1}^{(0)}, u_{3}^{(1)}$ and $\varphi^{(0)}$ may be taken as zero. If the piezoelectric constants $e_{26}$ and $\psi_{11}$ were absent, the first three of (9.1) would be the equations which were solved in reference [5] for the coupled thickness-shear, flexure and face-shear modes. The constant $e_{26}$ couples the thickness-shear deformation with the applied voltage; and $\psi_{11}$ couples the flexural deformation with the induced electric field.

The plate is bounded by $x_{1}= \pm l, x_{3}= \pm w$. The appropriate boundary conditions on $x_{1}= \pm i$ are

$$
T_{12}^{(0)}=T_{13}^{(0)}=T_{11}^{(1)}=D_{1}^{(1)}=0
$$

and we ignore the boundary conditions on $x_{\mathrm{s}}= \pm w$. Substitution of (4.17) in (9.2) converts the latter to: on $x_{1}= \pm l$,

$$
\begin{array}{ll}
2 h \kappa_{6} c_{66}\left(u_{2,1}^{(0)}+u_{1}^{(1)}\right)+2 h c_{56} u_{3,1}^{(0)}+e_{26} V=0, & \frac{2}{3} h^{3} \gamma_{11} u_{1,1}^{(1)}+\psi_{11} \varphi_{, 1}^{(1)}=0, \\
2 h \kappa_{6} c_{56}\left(u_{2,1}^{(0)}+u_{1}^{(1)}\right)+2 h c_{55} u_{3,1}^{(0)}+e_{25} V=0, & \frac{2}{3} h^{3} \psi_{11} u_{1,1}^{(1)}-\zeta_{11} \varphi_{, 1}^{(1)}=0 .
\end{array}
$$

A set of auxiliary functions which may be used to remove the inhomogeneous terms, $V$, from the electrostatic equation (9.1) $)_{4}$ and the boundary conditions (9.3), is $u_{2}^{(0) A}=\frac{1}{2} h^{-1} V P_{2} x_{1}, \quad u_{3}^{(0) A}=\frac{1}{2} h^{-1} V P_{3} x_{1}, \quad u_{1}^{(1) A}=0, \quad \varphi^{(1) A}=\frac{1}{3} h^{2} V$,

where

$$
P_{2}=\left(e_{25} c_{56}-e_{26} c_{55}\right) / \kappa_{6}\left(c_{55} c_{66}-c_{56}^{2}\right), \quad P_{3}=\left(e_{26} c_{56}-e_{25} c_{66}\right) /\left(c_{55} c_{66}-c_{56}^{2}\right)
$$

Then $G_{i}^{(0)}=G_{b}^{(1)}=0$ and the residual inhomogeneous differential equations are

$$
\begin{aligned}
& c_{68} K_{6}^{2}\left(u_{2,11}^{(0) R}+u_{1,1}^{(1) R}\right)+c_{56} \kappa_{6} u_{3,11}^{(0) R}+\rho \omega^{2} u_{2}^{(0) R}+\frac{1}{2} \rho \omega^{2} h^{-1} V P_{2} x_{1}=0, \\
& c_{56} K_{6}\left(u_{2,11}^{(0) R}+u_{1,1}^{(1) R}\right)+c_{55} \kappa_{6} u_{3,11}^{(0) R}+\rho \omega^{2} u_{3}^{(0) R}+\frac{1}{2} \rho \omega^{2} h^{-1} V P_{3} x_{1}=0,
\end{aligned}
$$

$\frac{2}{3} h^{3} \gamma_{11} u_{1,11}^{(1) R}-2 h \kappa_{6}^{2} c_{66}\left(u_{2,1}^{(0) R}+u_{1}^{(1) R}\right)-2 h \kappa_{6} c_{56} u_{3,1}^{(0) R}$

$$
\begin{aligned}
& +2 h \psi_{11} \varphi_{, 11}^{(1) R}+\frac{2}{3} \rho h^{3} \omega^{2} u_{1}^{(1) R}=0, \\
& \frac{2}{3} h^{3} \psi_{11} u_{1,11}^{(1) R}-\zeta_{11} \varphi_{, 11}^{(1) R}+15 h^{-2} \epsilon_{22}^{S} \varphi^{(1) R}=0 .
\end{aligned}
$$

In this case, we see that $(9.1)_{3}$ has also become homogeneous.

The boundary conditions to be satisfied by the residual solution are

$$
\begin{array}{ll}
2 h \kappa_{6} c_{66}\left(u_{2,1}^{(0) R}+u_{1}^{(1) R}\right)+2 h c_{56} u_{3,1}^{(0) R}=0, & \frac{2}{3} h^{3} \gamma_{11} u_{1,1}^{(1) R}+\psi_{11} \varphi_{1}^{(1) R}=0, \\
2 h \kappa_{6} c_{56}\left(u_{2,1}^{(0) R}+u_{1}^{(1) R}\right)+2 h c_{55} u_{3,1}^{(0) R}=0, & \frac{2}{3} h^{3} \psi_{11} u_{1,1}^{(1) R}-\zeta_{11} \varphi_{, 1}^{(1) R}=0 .
\end{array}
$$


We must now find the orthogonal functions for the series solution of the residual problem; i.e., we must find the solutions of (9.1) and (9.3) with $V=0$. Consider

$$
\left(u_{2}^{(0)}, u_{3}^{(0)}\right)=(A, B) \sin \xi x_{1}, \quad\left(u_{1}^{(1)}, \varphi^{(1)}\right)=(C, D) \cos \xi x_{1} .
$$

These satisfy (9.1), with $V=0$, if

$$
\begin{gathered}
A\left(\kappa_{6}^{2} c_{66} \xi^{2}-\rho \omega^{2}\right)+B \kappa_{6} c_{56} \xi^{2}+C \kappa_{6}^{2} c_{66} \xi=0, \\
A \kappa_{6} c_{56} \xi^{2}+B\left(c_{55} \xi^{2}-\rho \omega^{2}\right)+C \kappa_{6} c_{56} \xi=0, \\
A \kappa_{6}^{2} c_{66} \xi+B \kappa_{6} c_{56} \xi+C\left(\kappa_{6}^{2} c_{66}+\frac{1}{3} h^{2} \gamma_{11} \xi^{2}-\frac{1}{3} \rho h^{2} \omega^{2}\right)+D \frac{1}{2} h^{-1} \psi_{11} \xi^{2}=0, \\
C \psi_{11} \xi^{2}-D \frac{3}{7} h^{-3}\left(15 h^{-2} \epsilon_{22}^{S}+\zeta_{11} \xi^{2}\right)=0 ;
\end{gathered}
$$

For a non-trivial solution, the determinant of the coefficients of $A, B, C, D$ must vanish, leading to a quartic equation in $\xi^{2}$ as against a cubic when the piezoelectric constant, $\psi_{11}$, is zero. The additional root is imaginary for all $\omega$, i.e., it produces a non-propagating mode. Thus, there will be no additional resonances due to the piezoelectric effect. The remaining three roots are almost the same as those with $\psi_{11}=0$, due to the fact that $\psi_{11}^{2}$ is small in comparison with $\zeta_{11} \gamma_{11}$ and $\epsilon_{22}^{S} \gamma_{11}$. Hence there will be little effect on the wave numbers $\xi$ by computing all four roots from the determinant of $(9.8)$ with $\psi_{11}=0$. Thus we have $\xi_{n}, n=1,2,3$, as functions of $\omega^{2}$ from the first three of (9.8) and

$$
\xi_{4}^{2}=-15 \epsilon_{22}^{S} / \zeta_{11} h^{2},
$$

independent of $\omega$, from the fourth. Corresponding to $\xi_{n}, n=1,2$, 3, we may compute amplitude ratios

$$
p_{n}=A_{n} / C_{n}, \quad q_{n}=B_{n} / C_{n},
$$

from the first two of (9.8) and

$$
r_{n}=\epsilon_{22}^{S} D_{n} / \psi_{11} C_{n}=\frac{2}{3} h^{3} \epsilon_{22}^{S} \xi_{n}^{2} /\left(15 \epsilon_{22}^{S} h^{-2}+\zeta_{11} \xi_{n}^{2}\right),
$$

from the fourth. To the approximation (9.9), $A_{4}=B_{4}=C_{4}=0$. Consequently, we may write (9.7), with $\xi_{4}^{\prime} \equiv i \xi_{4}$, as

$$
\begin{gathered}
\left(u_{2}^{(0)}, u_{3}^{(0)}\right)=\sum_{n=1}^{3}\left(p_{n}, q_{n}\right) C_{n} \sin \xi_{n} x_{1}, \quad u_{1}^{(1)}=\sum_{n=1}^{3} C_{n} \cos \xi_{n} x_{1}, \\
\varphi^{(1)}=D_{4} \cosh \xi_{4}^{\prime} x_{1}+\left(\psi_{11} / \epsilon_{22}^{S}\right) \sum_{n=1}^{3} r_{n} C_{n} \cos \xi_{n} x_{1} .
\end{gathered}
$$

We now insert (9.12) in the boundary conditions (9.3), with $V=0$, and find

$$
\begin{gathered}
\sum_{n=1}^{3} C_{n}\left[\kappa_{6}^{2} c_{68}\left(p_{n} \xi_{n}+1\right)+\kappa_{6} c_{58} q_{n} \xi_{n}\right] \cos \xi_{n} l=0, \\
\sum_{n=1}^{3} C_{n}\left[\kappa_{6} C_{56}\left(p_{n} \xi_{n}+1\right)+c_{55} q_{n} \xi_{n}\right] \cos \xi_{n} l=0, \\
D_{4} \psi_{11} \epsilon_{22}^{S} \xi_{4}^{\prime} \sinh \xi_{4}^{\prime} l-\sum_{n=1}^{3} C_{n}\left(\frac{2}{3} h^{3} \gamma_{11} \epsilon_{22}^{S}+\psi_{11}^{2} r_{n}\right) \xi_{n} \sin \xi_{n} l=0, \\
D_{4} \zeta_{11} \epsilon_{22}^{S} \xi_{4}^{\prime} \sinh \xi_{4}^{\prime} l+\psi_{11} \sum_{n=1}^{3} C_{n}\left(\frac{2}{3} h^{3} \epsilon_{22}^{S}-\zeta_{11} r_{n}\right) \xi_{n} \sin \xi_{n} l=0 .
\end{gathered}
$$


Elimination of $C_{n}$ and $D_{4}$ from (9.13) results in a transcendental frequency equation relating $l / h$ to the frequency. For a given $l / h$, the frequency equation has an infinity of roots, $\omega_{\mu}$, to each of which there correspond amplitude ratios $p_{n}^{\mu}, q_{n}^{\mu}, r_{n}^{\mu}$, from (9.10) and (9.11), and amplitude ratios

$$
\beta_{n}=C_{n} / C_{3}, \quad n=1,2 ; \quad \beta_{3}=1 ; \quad \beta_{4}=D_{4} / C_{3}
$$

obtained from (9.13). Inserting these results in (9.12), we obtain the expressions for the orthogonal functions:

$$
\begin{gathered}
\left(u_{2}^{(0) \mu}, u_{3}^{(0) \mu}\right)=\sum_{n=1}^{3}\left(p_{n}^{\mu}, q_{n}^{\mu}\right) \beta_{n}^{\mu} \sin \xi_{n}^{\mu} x_{1}, \quad u_{1}^{(1) \mu}=\sum_{n=1}^{3} \beta_{n}^{\mu} \cos \xi_{n}^{\mu} x_{1}, \\
\varphi^{(1) \mu}=\beta_{4}^{\mu} \cosh \xi_{4}^{\prime} x_{1}+\left(\psi_{11} / \epsilon_{22}^{S}\right) \sum_{n=1}^{3} r_{n}^{\mu} \beta_{n}^{\mu} \cos \xi_{n}^{\mu} x_{1},
\end{gathered}
$$

in which the common factor $C_{3}$ has been set equal to unity.

Little error in a computation of frequencies and amplitude ratios will result if the frequency equation is obtained from the first three of (9.13) with $D_{4}=0$. The frequency equation would then have the same form as that in reference [5] but the material constants in the coefficients of the transcendental functions would have slightly different values due to the presence of the piezoelectric constants. In such an approximation the amplitude ratios $\beta_{1}^{\mu}$ and $\beta_{2}^{\mu}$ would be obtained from the first two of (9.13) and $\beta_{4}^{\mu}$ from the fourth.

The normalization factor is found by inserting (9.15) in (6.2), remembering that $u_{1}^{(0) \mu}=u_{3}^{(1) \mu}=0$, with the result

$$
N_{(\mu)}=\frac{1}{2} A \sum_{m=1}^{3} \sum_{n=1}^{3} \beta_{m}^{\mu} \beta_{n}^{\mu}\left[\left(p_{m}^{\mu} p_{n}^{\mu}+q_{m}^{\mu} q_{n}^{\mu}+\frac{1}{3} h^{2}\right) \mathcal{S}_{m n}^{-}-\left(p_{m}^{\mu} p_{n}^{\mu}+q_{m}^{\mu} q_{n}^{\mu}-\frac{1}{3} h^{2}\right) \mathcal{S}_{m n}^{+}\right],
$$

where $S_{m n}^{ \pm}=\left(\xi_{m}^{\mu} l \pm \xi_{n}^{\mu} l\right)^{-1} \sin \left(\xi_{m}^{\mu} l \pm \xi_{n}^{\mu} l\right)$. Then, from (7.18) $)_{1}$, we have $B_{\mu}=0$ since $G_{i}^{(0)}=G_{b}^{(1)}=0$; and, from $(7.18)_{2}$, noting that $u_{1}^{(0) A}, u_{1}^{(1) A}$ and $u_{3}^{(1) A}$ are also zero,

$$
C_{\mu}=\frac{1}{2} A N_{(\mu)}^{-1} \sum_{n=1}^{3}\left(P_{2} p_{n}^{\mu}+P_{3} q_{n}^{\mu}\right)\left(\beta_{n}^{\mu} / \xi_{n}^{\mu} h\right)\left[\left(\xi_{n}^{\mu} l\right)^{-1} \sin \xi_{n}^{\mu} l-\cos \xi_{n}^{\mu} l\right] .
$$

Finally, from (7.19),

$$
A_{\mu}=C_{\mu} \omega^{2} /\left(\omega_{\mu}^{2}-\omega^{2}\right) .
$$

When (9.18), (9.15) and (9.4) are substituted in (7.20) the solution is completed except for a formula for the correction factor $\kappa_{6}$. This is found by equating the frequency of free thickness-shear vibrations, from $(9.1)_{3}$, with Lawson's analogous solution [7] of the three-dimensional equations. The result, as obtained previously [2], is

$$
\kappa_{8}^{2}=\frac{1}{3} \alpha_{0}^{2}\left(1+e_{26}^{2} / \epsilon_{22}^{S} C_{66}^{E}\right),
$$

where $\alpha_{0}$ is the lowest, non-zero root of

$$
\tan \alpha=\alpha\left(1+\underset{\epsilon_{22} C}{S} C_{B 6}^{E} / e_{28}^{2}\right)
$$

The formula (8.4) for surface charge requires expressions for $Y_{\mu}$ and $L$, in addition 
to $A_{\mu}$. These are obtained by substituting (9.15) and (9.4) in (8.5) and (8.6), respectively, with the results:

$$
\begin{gathered}
Y_{\mu}=A \sum_{n=1}^{3}\left[\kappa_{6} e_{26}\left(p_{n}^{\mu} \xi_{n}^{\mu}+1\right)+e_{25} q_{n}^{\mu} \xi_{n}^{\mu}-\left(15 / 4 h^{3}\right) \psi_{11} r_{n}^{\mu}\right]\left(\beta_{n}^{\mu} / \xi_{n}^{\mu} l\right) \sin \xi_{n}^{\mu} l \\
-A\left(15 / 4 h^{3}\right) \epsilon_{22}^{S} \beta_{4}^{\mu}\left(\xi_{4}^{\prime} l\right)^{-1} \sinh \xi_{4}^{\prime} l, \\
L=\frac{1}{2} A h^{-1}\left(\kappa_{6} e_{26} P_{2}+e_{25} P_{3}-[5 / 2] \epsilon_{22}^{S}\right),
\end{gathered}
$$

The expression (9.18) for $A_{\mu}$ has, of course, the term $\omega_{\mu}^{2}-\omega^{2}$ in the denominator. Hence, near a resonance only one term of the infinite series in the formula (8.4), for the surface charge $Q$, need be computed. We have suggested that, in computing the roots of the equations obtained by setting the determinants of the coefficients of (9.8) and (9.13) equal to zero, certain of the terms having $\psi_{11}$ as a factor be neglected. One would thereby take into account the coupling between the applied voltage and the strain, through $e_{26}$, and the generation of the induced electric field by the curvature, through the amplitude ratios $r_{n}$; but a part of the small counter effect of the induced electric field on the strain would be neglected. This approximation need not be made if adequate facilities are available for computing the roots of the quartic equation obtained from (9.8) and the complete $4 \times 4$ transcendental determinantal equation which would be obtained from the equations which would replace (9.13).

\section{REFERENCES}

1. R. D. Mindlin, High frequency vibrations of crystal plates, Quart. Appl. Math. 19, 51-61 (1961)

2. R. D. Mindlin, Forced thickness-shear and flexural vibrations of piezoelectric crystal plates, J. Appl. Phys. 23, 83-88 (1952)

3. W. P. Mason, Piezoelectric crystals and their application to ultrasonics, D. Van Nostrand Co., New York, 1950

4. R. Courant and D. Hilbert, Methods of Mathematical Physics, Interscience Publishers, New York

5. R. D. Mindlin and D. C. Gazis, Strong resonances of rectangular AT cut quartz plates. (Forthcoming)

6. I. Koga and H. Fukuyo, Vibration of thin piezoelectric quartz crystal plates, especially an $R_{1}-c u t$ ( $A T$-cut) rectangular plate, J. Inst. Elec. Comm. Engrs. of Japan 36, 59-67 (1953)

7. A. W. Lawson, The vibration of piezoelectric plates, Phys. Rev. 62, 71-75 (1942) 